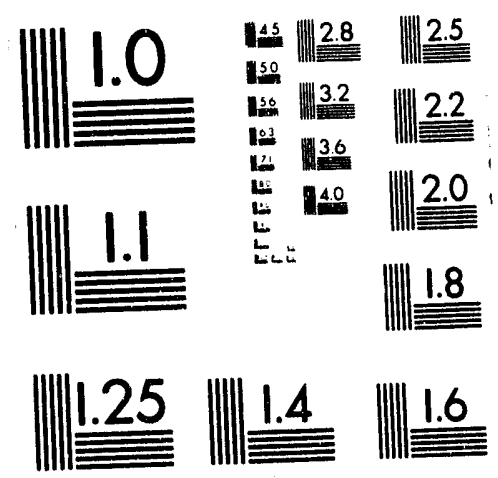



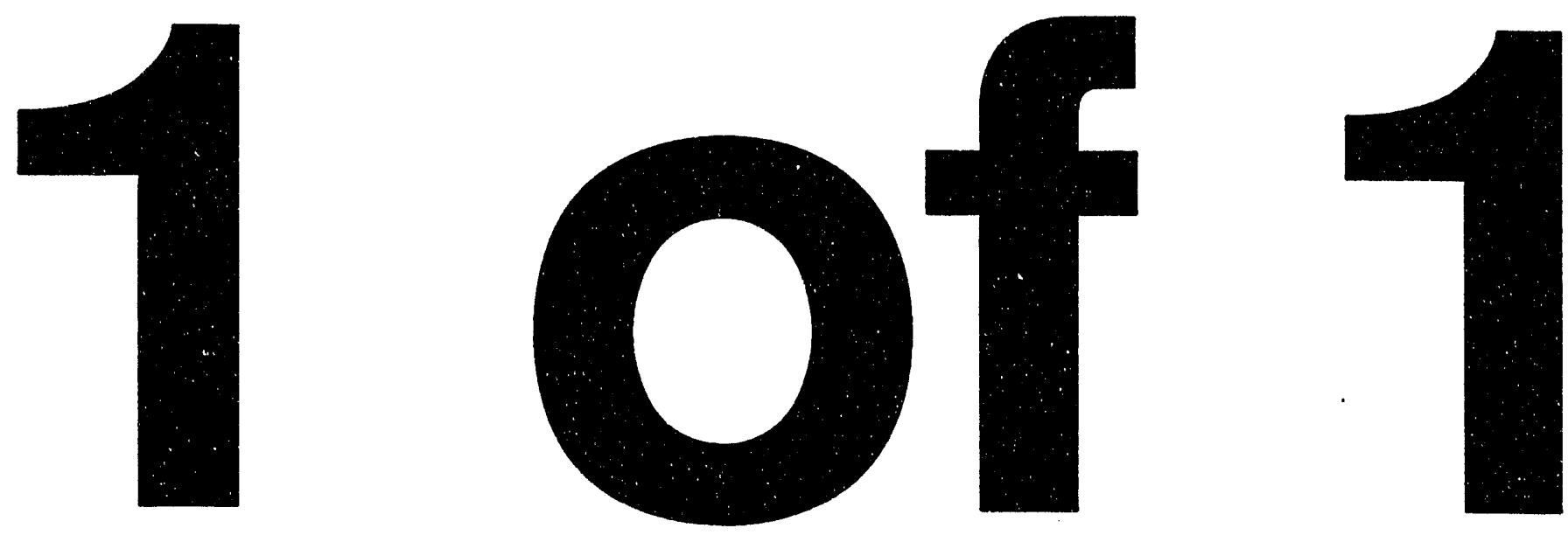


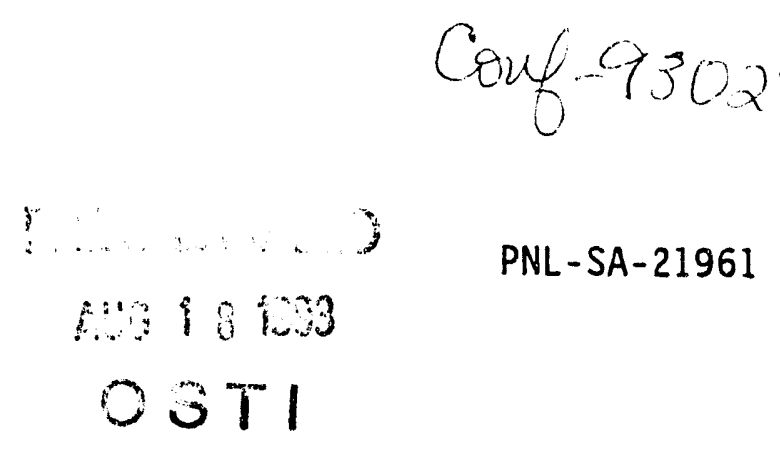

FOURIER TRANSFORM ION CYCLOTRON

RESONANCE VERSUS TIME OF FLIGHT

FOR PRECISION MASS MEASUREMENTS

R. T. Kouzes

\section{DISCLAIMER}

February 1993

This report was prepared as an account of work sponsored by an agency of the United States Government. Neither the United States Government nor any agency thereof, nor any of their employees, makes any warranty, express or implied, or assumes any legal liability or responsibility for the accuracy, completeness, or usefulness of any information, apparatus, product, or process disclosed, or represents that its use would not infringe privately owned rights. Reference herein to any specific commercial product, process, or service by trade name, trademark, manufacturer, or otherwise does not necessarily constitute or imply its endorsement, recommendation, or favoritg by the United States Government or any agency thereof. The views and opinions of authors expressed herein do not necessarily state or reflect those of the United States Government or any agency thereof.

Presented at the

Workshop on Traps for Antimatter

and Radioactive Nuclei

February 25-27, 1993

Vancouver, British Columbia

Prepared for

the U.S. Department of Energy

Contract DE-AC06-76RLO 1830

Pacific Northwest Laboratory Richland, Washington 99352 


\title{
Fourier Transform Ion Cyclotron Resonance Versus Time of Flight for Precision Mass Measurements
}

\author{
Richard T. Kouzes \\ Pacific Northwest Laboratory, † MS K1-87, PO Box 999, Richland, W A 99352
}

Abstract: Both Fourier Transform Ion Cyclotron Resonance and ICR Time-of-Flight mass spectroscopy have been applied to precision atomic mass measurements. This paper reviews the status of these approaches and compares their limitations.

\section{Introduction}

Traditional techniques for precision atomic mass measurements, which have relied on spectrometers with magnetic or crossed electric and magnetic fields, have been in use for many years, [1] and are still providing high precision mass values, such as the recent $0.32 \mathrm{keV}$ accuracy result for the ${ }^{76} \mathrm{Ge}-{ }^{76} \mathrm{Se}$ mass difference, [2] but typically give resolution in the range of parts in 105. Traditional time-of-flight mass spectroscopy (TOF-MS), based on the traversal of a known distance by ions of a known energy, is a cost effective mass spectroscopy tool, which also has a long history and is extensively used in chemistry and physics, [3] with resolution around the part in 5000 regime. Paul traps, ${ }^{[4]}$ which use an RF trapping field in a cell and do not require a magnetic field, is another popular commercial instrument with resolution of approximately a part in 104.

Ion Cyclotron Resonance Mass Spectroscopy (ICR-MS), a technique for precise measurements of ion masses based on the constancy of an ion's cyclotron frequency in a fixed magnetic field, started with the pioneering work by S.A. Goudsmit[5] and by L.G. Smith,[6] who obtained resolution of a part in 200,000 . Some of the first ultra-high accuracy atomic mass measurements with ICR-MS were carried out on the ${ }^{3} \mathrm{He}$-tritium mass pair, motivated by weak interaction physics applications. [7-9] The most impressive ICR-MS results are those with a precision of parts in 1010 in the mass range of $14-40$ amu.[10] Ion cyclotron resonance has been enhanced in recent years by the use of Fourier transform analysis of large transient recorded data records, giving rise to Fourier Transform Ion Cyclotron Resonance Mass Spectroscopy (FTICR-MS).[11,12] Most recent efforts in FTICR-MS have concentrated on developments for measuring organic compounds. A combination of ICR and TOF, which will hereafter be referred to as ICR-TOFMS, has recently produced a number of high resolution results on unstable nuclei at CERN. [13-17] This paper will make some comparisons of FTICR-MS and ICR-TOF-MS for application to precision atomic mass measurements of stable and unstable nuclei, where the relavent scale is an accuracy of $1 \mathrm{keV}$ (about 1 part in 108) and where halflives are longer than 10 milliseconds (optimistically). The atomic mass table is built up from mass chains, and ICR-MS brings a method of producing new types of mass chains to the mass measurement arena.

\section{Fourier Transform Ion Cyclotron Resonance Mass Spectroscopy}

Figure 1 shows schematically the process of FTICR-MS, starting from ion production, injection, and trapping through RF excitation, detection, and subsequent production of the mass spectrum from the transient. A typical FTICR-MS system, seen schematically in Figure 2, consists of a high vacuum system with a Penning trap [18] cell that traps ions axially via an electrostatic potential, and a solenoid magnetic field that constrains the ions radially. There are about 130 FTICR-MS systems in use, almost entirely in chemistry labs. Ions can be produced directly in the ICR cell with a filament ionizing a gas leaked into the vacuum system, or ions can be injected from an 

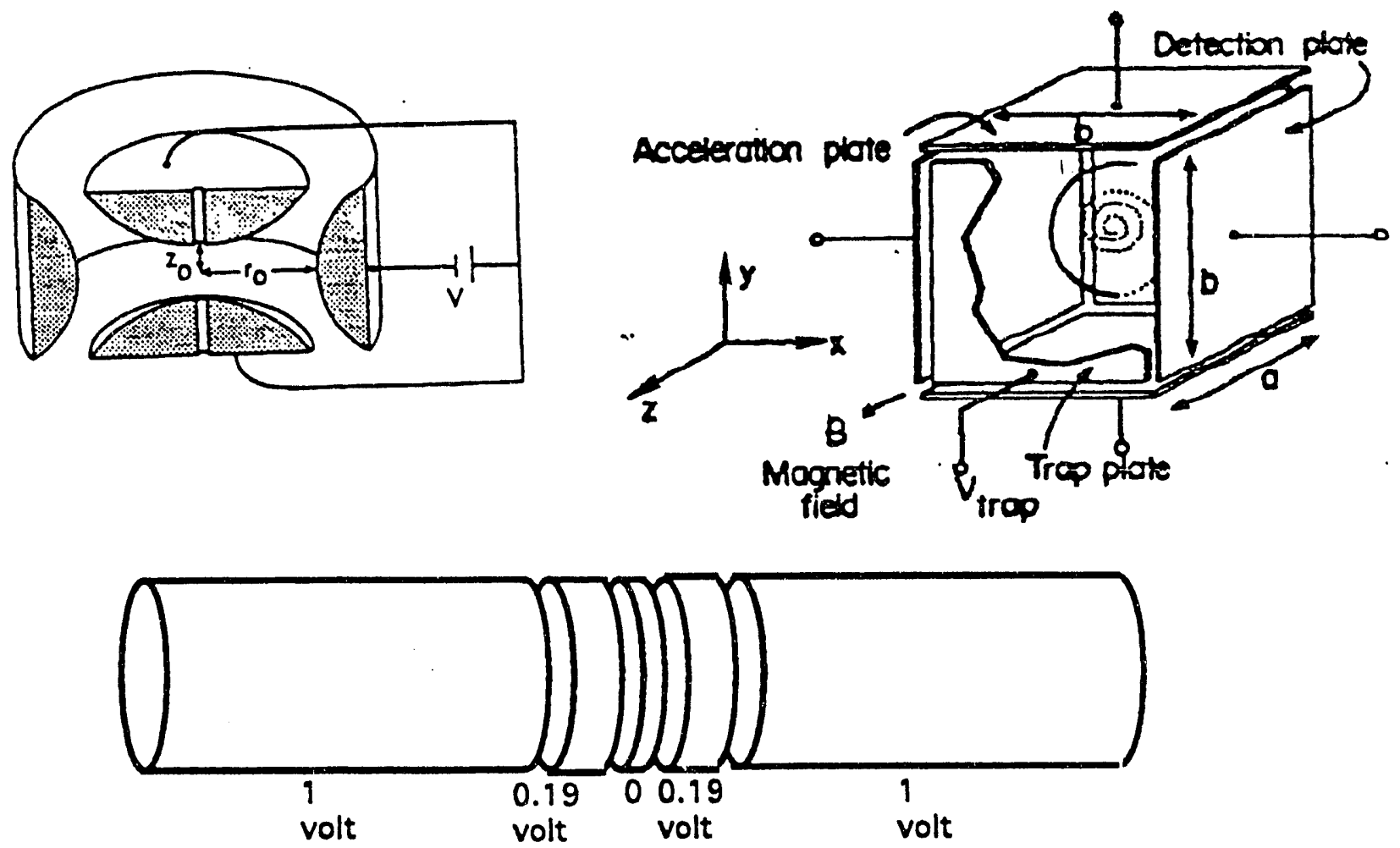

Figure 3: ICR cells can be traditional hyperbolic traps, [25] cylindrical quadratic traps, [26] or more commonly, a rectangular cells. [20]

external source. A wide range of external sources have been used, including electron bombardment, laser ablation, fast ion bombardment, fission fragment ionization, and electrospray, to name a few. The externally produced ions are transported into the cell in the solenoid field using electrostatic lenses or RF quadrupoles. The cell can be designed to have a quadratic electric potential, a shape traditionally associated with Penning traps, or can be a simple rectangular shape, as shown in Figure 3. The rectangular cell is most widely used in FTICR-MS systems because of its ease of construction for large volumes, and its performance for those interested in measuring masses over a broad mass range (up to $30,000 \mathrm{amu}$ or higher) with resolving power up to several million. We have built one of the cylindrical quadratic traps[26] at PNL for use in our 7 tesla FTICR-MS system.

The ions are captured in the cell by electrode gating, axial deflection, or collisions with a cooling gas. The ions might then be cooled via collisions to bunch them in the electrostatic well, a technique essential for obtaining good resolution for heavy molecules. The cell is divided asymuthally into four or more sectors for excitation and detection of the ion motion. If an RF field near the ion's cyclotron frequency is placed across the cell, the ions will be accelerated in a spiral orbit to larger radius. Typical FTICR-MS applications do not use this narrow band excitation, but rather use an RF "chirp" across the side plates of the cell to accelerate the ion bunch, thereby exciting a selectable broad mass range simultaneously. At this point in the FTICR-MS process, an ion bunch is now orbiting in the cell at some radius, and a signal is induced on the other two side plates of the cell near the ion's cyclotron frequency. This induced signal is amplified and detected either directly or heterodyned to a lower frequency. This analog signal, which is a sine wave for a single ion mass, is transient recorded at a sampling rate above the Nyquist frequency of the oscillation.

The resulting digital record of the induced signal is Fourier transformed to give a mass spectrum. 
The spectrum is usually calibrated with known masses using a function that is second order in the inverse frequency. One feature of FTICR-MS that has an impact on its applicability to precision atomic mass measurements, is it can produce a spectrum of many masses simultaneously, allowing a comparison without systematic effects arising from sequential measurements. We will see what limits this feature later in this paper. There are now about 100 FTICR-MS systems in use worldwide.

A FTICR-MS system with very high mass accuracy and mass resolution, developed at the American Cyanamid Company, uses an external source to inject ions through an RF quadrupole mass filter and then through a set of electrostatic lenses into an ICR cell at the center of a 7 tesla magnetic field.[19] This signal is sampled and digitized at rates from $250 \mathrm{kHz}$ to $4 \mathrm{MHz}$, accumulating up to 524,288 samples. The Cyanamid group has obtained mass accuracy as good as a few parts in $10^{8}$ for masses from 60 to $1000 \mathrm{amu}$. A measurement obtained with this FTICRMS for five isotopes of natural abundance xenon gave values deviating by about 5 parts in $10^{8}$ from the tabled values for the xenon isotopic masses, only slightly worse than the present errors for some of these masses, while indicating a significant error of $27 \mathrm{keV}$ in the ${ }^{134} \mathrm{Xe}$ mass. [20] The xenon spectrum from a single FTICR-MS measurement is seen in Figure 4.

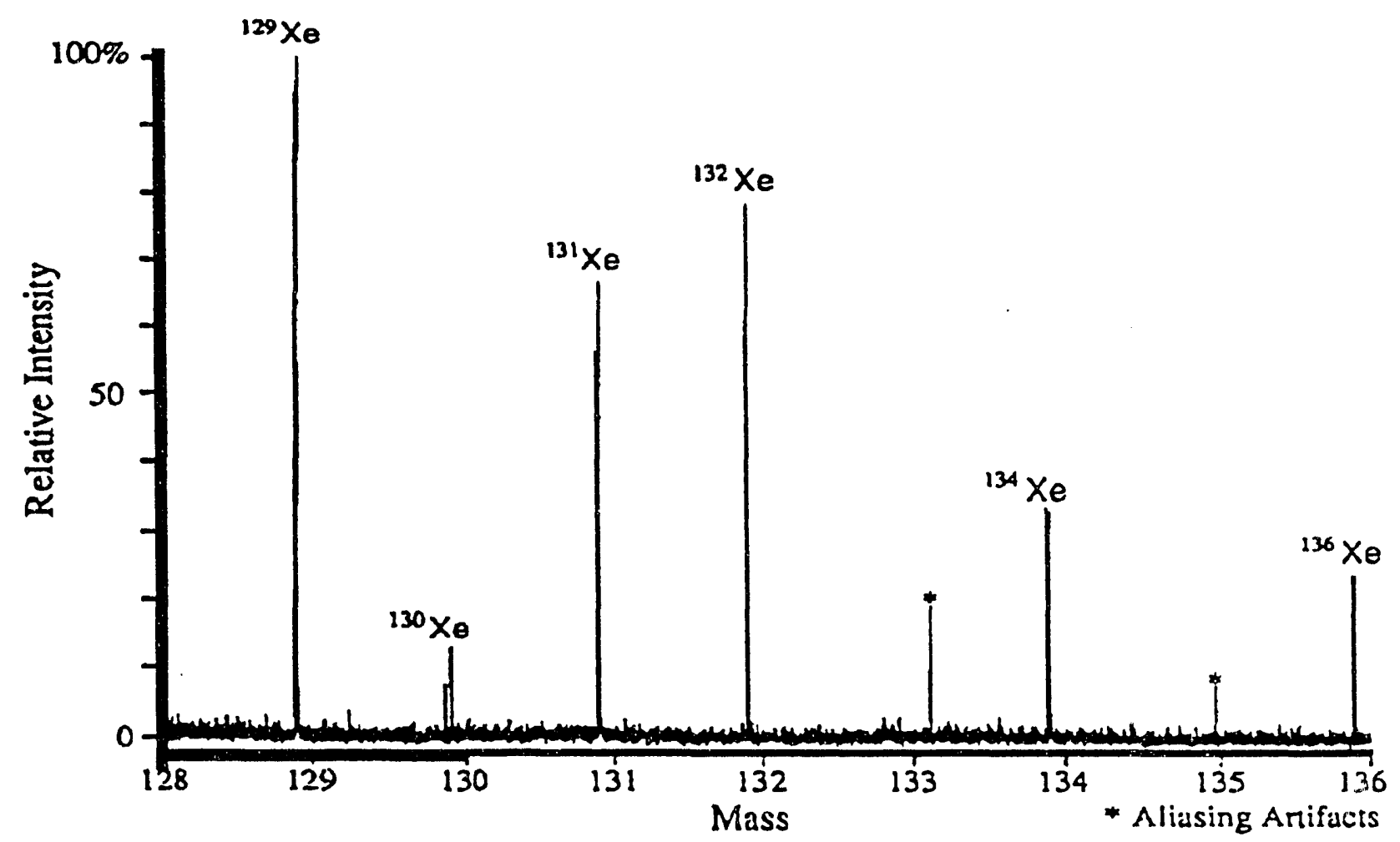

Figure 4: FTICR-MS spectrum of the xenon isotopes.[20]

The Molecular Science Research Center at the Pacific Northwest Laboratory (PNL) has installed a similar 7 tesla FTICR-MS built by IonSpec, and we are designing 3 tesla and 12 tesla FTICR-MS systems. The present 7 tesla device has produced transients as long as 90 seconds for very heavy molecules.[21] The main purpose of these systems is for organic chemistry work, but one of the, applications planned for these FTICR-MS systems will be to carry out precision atomic mass measurements. The desire is to obtain mass measurements at the $1 \mathrm{keV}$ to $100 \mathrm{eV}$ level of accuracy (better than 1 part in 108). Limitations to the precision of such measurements have been evaluated 
and will impact the development of these systems.[22] One area of development at PNL is in improved data systems for FTICR-MS. We are assembling a data system capable of capturing and processing transients of up to 16 megawords, a requirement for obtaining high resolution measurements for transients lasting up to several minutes.

Other recent work on FTICR-MS measurements of atomic masses include a six second transient of xenon, [24] and measurements on oxygen with a resolving power of 300 million.[24]

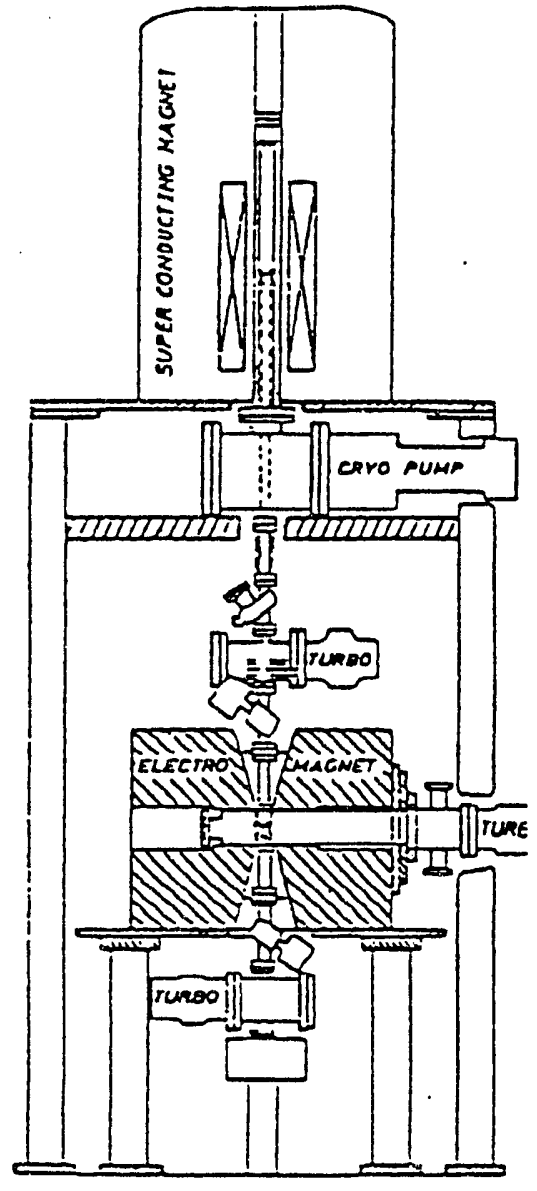

Figure 5: ICR-TOF-MS apparatus at CERN.[14]

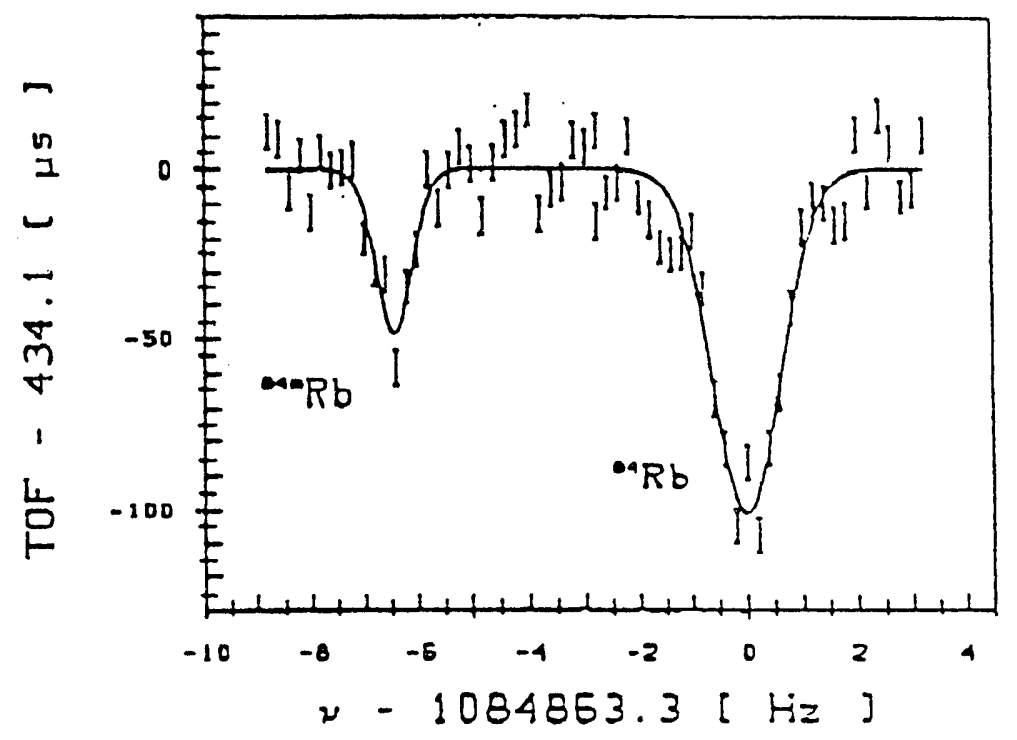

Figure 6: ICR-TOF-MS spectrum.[28]

\section{Ion Cyclotron Resonance Time of Flight Mass Spectroscopy}

In the ICR-TOF-MS system, the mass determination is also made through a measurement of the cyclotron resonance condition, but this is determined in a different fashion than in FIICR-MS. Figure 5 shows schematically the ICR-TOF-MS apparatus. In this scheme, ions are first accumulated into a bunching cell and then are injected in to a hyperbolic trap where they are subject to a narrow band RF excitation.

After acceleration, the ions are ejected axially out of the trap, and their time of flight to a microchannel detector is measured. The solenoid magnetic field strength decreases in tice flight path to the detector. If the ions were on resonance with the exciting RF field, their orbital energy in the trap increased, and as the ions traverse the magnetic field gradient in the flight path to the 
detector, their axial velocity increases. The change in flight time is the observable resulting from ions on resonance. Thus, as ions are injected, accelerated, and detected as a function of exciting $\mathrm{RF}$ frequency, the resonance frequency can be determined. A measurement takes about 15 minutes.

Figure 6 shows a spectrum resulting from this technique. Single ion sensitivity is possible, with 50 ions at a time as a typical bunch. This allows for experiments on short-lived radioactive nuclei, such as the measurement of ${ }^{124} \mathrm{Cs}$ with a halflife of $30.8 \mathrm{sec}$.

\section{Resolution, Precision and Accuracy}

There can be some confusion about the distinction between the terms resolution, precision, and accuracy for mass measurements. Resolution is a measure of the width of the spectral feature associated with a mass, and is equal to the FWHM $(\Delta \mathrm{m})$ of a peak divicied by the mass $(\mathrm{m})$. Resolving power is the inverse of the resolution value.

The precision of a mass measurement $(\delta \mathrm{m})$ is how well the centroid of a spectral feature can be found. The precision of a measurement is usually much better than the resolution (typically by anywhere from one to six orders of magnitude). It is limited by statistics, signal to noise, and knowledge of the peak shape.

The accuracy is the parameter of interest when making a mass determination, and is the precision with which a value can be measured relative to another mass. The absolute accuracy is the precision relative to some absolute scale, ${ }^{12} \mathrm{C}$ and the atomic mass unit in this case. Confusion is often produced by the terminology since various authors will use any or all of these measures of system performance.

\section{Some Ion Cyclotron Resonance Theory}

Ion cyclotron resonance is based on very simple physics, but is subject to a number of complications. An ion of mass (m) and charge ( $\mathrm{z}$ ) in a uniform magnetic field $(\mathrm{B})$ has an angular cyclotron frequency $\left(\omega_{c}\right)$ given by

$$
\omega_{c}=z B / m
$$

For atoms this is a frequency $\left(v_{c}\right)$ of the order of a $\mathrm{MHz}$.

When an ion is placed in an electrostatic trap along the axial direction of potential $V$, the ion oscillates axially, and the observed radial motion is modified. If the trap potential is quadratic, the ions axial motion is harmonic with an angular frequency $\left(\omega_{2}\right)$ given by[25]

$$
\omega_{z}=\left(4 \mathrm{zG}_{\mathrm{z}} \mathrm{V} / \mathrm{m}\right)^{1 / 2}
$$

where $G_{z}$ is a geometry factor of order unity. For atoms, this axial frequency $\left(v_{z}\right)$ ranges from one $\mathrm{kHz}$ to hundreds of $\mathrm{kHz}$ depending on the trap geometry. The ions have an angular motion close to their cyclotron frequency superimposed on a slow precession of the orbit ai the magnetron angular frequency $\left(\omega_{\mathrm{m}}\right)$. There is some confusion as to the exact definition used for the magnetron frequency. One definition that is used has no mass dependence and is given by [25]

$$
\omega_{\mathrm{m}} \approx 2 \mathrm{GV} / \mathrm{B} \text {. }
$$

This angular magnetron frequency corresponds to a frequency $\left(v_{m}\right)$ for atoms ranging from $\mathrm{Hz}$ to $\mathrm{kHz}$ depending on the trap geometry. The definition used here for the exact magnetron frequency 
value, with a slight mass dependence, is given by the equations of motion.

The actual angular motion eigenfrequencies for an ion in a quadratic trap are given by[25]

$$
\omega_{ \pm}=\omega_{c} / 2\left[1 \pm\left(1-2 \omega_{z}^{2} / \omega_{c}^{2}\right)^{1 / 2}\right] .
$$

These values are equivalent to

$$
\omega_{+}=\omega_{c}-\omega_{m} \text { and } \omega_{-}=\omega_{m} .
$$

The ion motion is thus complex, and precision mass measurements require understanding and controlling the effects that shift the observed frequencies. Figure 7 shows the motion schematically for an ion in a Penning trap.

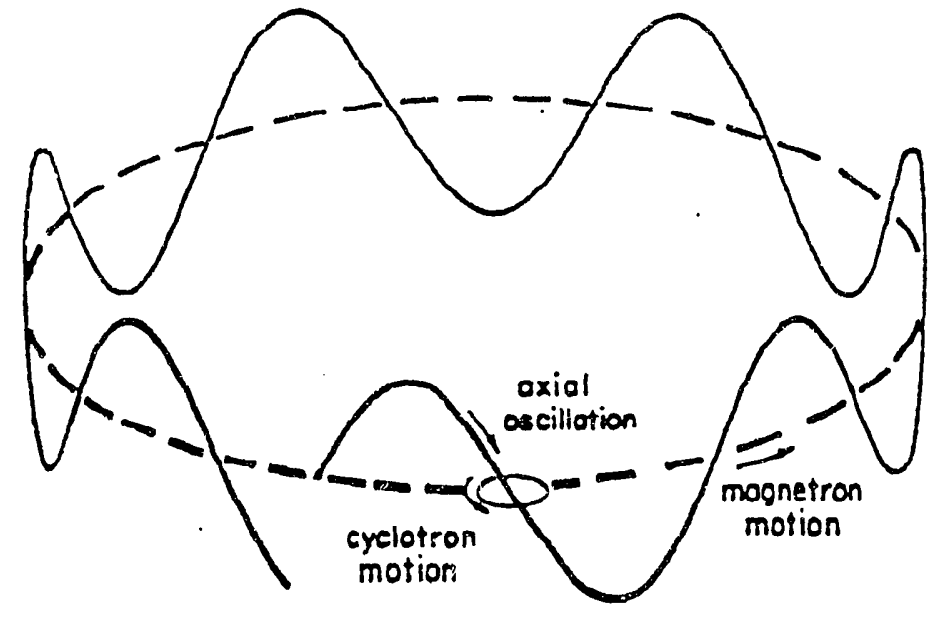

Figure 7: Ion motion in a trap is a combination of axial, cyclotron and magnetron motion.[27]

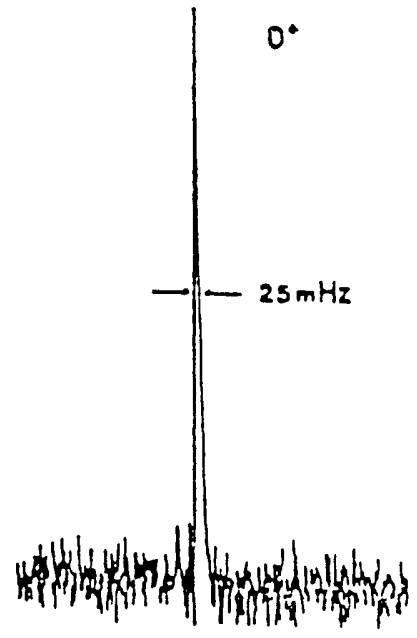

Figure 8: High resolution FTICR-MS measurement of oxygen with resolving power of 300 million. [24]

For a frequency measurement such as FTICR-MS and ICR-TOF-MS the resolution is constrained by the Fourier limit, atising from the Schwarz inequality, which implies that for a measurement or a signal lasting a time $\tau$, the resolving power is limited to

$$
\mathrm{m} / \Delta \mathrm{m} \equiv \omega / \Delta \omega \equiv v / \Delta v \leq 2.5 \times 10^{7} \mathrm{zB} \tau / \mathrm{m}
$$

where $\mathrm{B}$ is in tesla, $\mathrm{m}$ is in amu and $\mathrm{z}$ is in units of electron charges. Figure 8 shows an example of an FTICR-MS measurement of oxygen with a resolving power of 300 million.[24]

\section{Reality}

There are a number of factors that limit the resolution of an FTICR-MS instrument for precision atomic mass measurements. These include total sample size (transient time length), $A D C$ sampling rate and resolution, several effects contributing to signal decay in time, relativistic effects, space charge effects, and initial spacial and energy spreads. 
To obtain the level of resolution desired for precision measurements, transients must survive and be recorded for a time of about 10 seconds. For a 7 tesla magnet, a 10 second transient limits the resolution of a mass 100 amu ion to no better than about one part in $10^{7}$, which should provide a precision of better than a part in $10^{8}$, or about $1 \mathrm{keV}$. Decay of the signal arises from a number of factors including residual gas collisions (requiring vacuums of $10^{-9}$ torr), dephasing due to electric and magnetic field inhomogeneities, energy dissipation in the external circuit, and collective effects. Electric and magnetic field inhomogeneities cause the loss of ions to the walls as well as causing the ions to spread in phase, with a resulting signal decay. Energy dissipation in the external circuit can be a significant limit to signal lifetime, including a linear dependence of decay time on the total ion cloud charge. Collective effects through Coulomb interactions not only cause signal decay, but also produce frequency shifts.

The observed angular frequency $\omega_{+}$is shifted slightly from $\omega_{c}$ by a mass dependent term. In nonquadratic traps, the shift is not fixed, since the motion is nonharmonic, and must be minimized by using small potentials or novel cell geometries. Most difficult to control are collective effects that vary with ion density and thus from measurement to measurement. Collective effects become especially important for obtaining a resolution greater than about a ppm. It is $\omega_{+}$which is observed in FTICR-MS, as opposed to $\omega_{c}$ in ICR-TOF-MS.

These same effects have consequence for ICR-TOF-MS, since the resolution is determined largely by the same Fourier limit, except that it is the excite time rather than the detect time that is relevant. Since the measurement depends upon a sequence of TOF values as a function of excite frequency, the reproducability of conditions from shot to shot is critical, especially the magnetic field.

\section{Comparisons}

How do FTICR-MS and ICR-TOF-MS compare for precision atomic mass measurements? They share similar ion injection and trapping features. The limitations produced by the trapping cell and magnetic field on the device resolution is similar in both cases.

The detection techniques differ, with different limitations imposed by each. FTICR-MS typically requires thousands of ions to produce an adequate signal to noise, which linnits sensitivity for rare species such as radioactive nuclei and produces observed mass shifts due to collective effects. Few ion sensitivity with broadband detection is an area of development for FTICR-MS. With improved sensitivity, the collective effects will be reduced. ICR-TOF-MS in principle has single ion sensitivity since detection is via channelplate amplification, and is thus perhaps better suited for few ion measurements. The orbit radii for various ions in an FIICR-MS system may vary which can introduce shifts and broadening.

FTICR-MS makes a complete measurement of the mass or masses of interest simultaneously, however, collective effects must be considered, especially above the part in $10^{7}$ resolution level. Complete measurements can be made in a short time period (a few seconds), which allows FIICRMS to be combined with gas or liquid chromotography, a feature of importance to chemists. This same feature is potentially of value for radioactive nuclei. ICR-TOF-MS requires a sequence of measurements to map out the resonance, requiring stability of the system over this time of several minutes. This makes ICR-TOF-MS more sensitive to systematic effects from shot to shot, especially the collective effects due to the varying number of ions in the trap on each measurement. The advantage of ease of measurement with FTICR-MS will make it the method of choice in experiments where samples are not limited to a few ions.

Resolution is determined by the time for excitation in ICR-TOF-MS and by the time for detection in FTICR-MS. It is perhaps easier to detect ions for a longer time than it is to excite them with a 
low intensity RF signal for this time, an advantage for FTICR-MS.

The design of the cell has an impact on measurements in both FTICR-MS and ICR-TOF-MS. For the quality traps needed for the high-precision measurements, quadratic potentials are needed. New open ended cells with quadratic potentials not only provide greater access to the trap, but also reduce the perturbations found in hyperbolic geometry traps due to effects such as holes in the end caps.[26] These cell geometries will find use in both FIICR-MS and ICR-TOF-MS.

The cost and maintenance of the system must also be considered. There are three commercial companies that sell FTICR-MS systems with resolution of better than a part in a million. These systems cost about half a million dollars and require little routine maintenance. ICR-TOF-MS systems are not commercially available.

tPacific Northwest Laboratory is operated for the U.S. Department of Energy by Battelle Memorial Institute under contract DE-AC06-76RLO 1830.

\section{References}

[1] J.J. Thomson, Nature 86 (1911) 468.

[2] J.G. Hykawy, et. al., Phys. Rev Lettt 67 (1991) 1708.

[3] D.J. Viera et. al., Phys. Rev. Lett. 57 (1986) 3253.

[4] W. Paul, Rev. Mod. Phys. 62 (1990) 531.

[5] S.A. Goudsmit, Phys. Rev. 74 (1948) 622.

[6] L.G. Smith and C.C. Damm, Rev. Sci. Inst. 27 (1956) 638.

[7] E.F. Lippmaa, et. al. 1984, JETP Lett. 39, 647.

[8] E.N. Nikolaev, et. al. 1984, JETP Lett. 39, 535.

[9] V L Talrose and E.N. Nikolaev 1986, Adv. Mass Spect. 1985, edited by J.F.J. Todd, J. Wiley and Sons.

[10] E.A. Cornell et.al., Phys. Rev. Lett. 63 (1989) 1674.

[11] M.B. Comisarow and A.G. Marshall, J. Chem. Phys. 69 (1978) 4097.

[12] M. Allemann, et. al., Int. Journal of Mass Spectrometry and Ion Physics 46 (1983) 139.

[13] G. Graff, H. Kalinowsky, J. Traut, Z. Phys. A297 (1980) 35.

[14] P. Dabkiewicz, et. al., Proc. 7th Conf. on Atomic Masses and Fund. Const., AMCO-7, Darmstadt-Seeheiri, ed. O. Klepper (1984) 684.

[15] H. Schnatz et. al., Nucl. inst. and Meth. A251 (1986) 17.

[16] H. Stolzenberg, et. al., Phys. Rev. Lett. 65 (1990) 3104.

[17] St. Becker et. al., Int. J. Mass Spect. Ion Proc. 99 (1990) 53.

[18] F.M. Penning, Physica 3 (1936) 873.

[19] J.T. Meek, W G Millen, T J Francl, G W Stockton, M L Thomson, R S Wayne, Proceedings 35th ASMS Conference on Mass Spectrometry and Allied Topics, Denver (1987) 1122.

[20] J.T. Meek, W G Millen, G W Stockton, R T Kouzes, Phys. Rev. C41 (1990) 2921.

[21] B. Winger et. al., submitted to JASMS (January, 1993).

[22] L.K. Herold, R T Kouzes 1990, Int. J. Mass Spect. Ion Proc. 96, 275.

[23] C.B. Lebrilla et. al., Int. J. Mass Spect. and Ion Proc. 87 (1989) R7.

[24] M. Bamberg, M. Allemann, K.P. Wanczek, Proceedings 35th ASMS Conference on Mass Spectrometry and Allied Topics, Denver (May 24-29, 1987) 1116.

[25] J.B. Jeffries, S.E. Barlow, G.H. Dunn, Int. J. Mass Spect. and Ion Proc. 54 (1983) 169.

[26] G. Gabrielse, L. Haarsma, S.L. Rolston, Int. J. Mass Spect. and Ion Proc. 88 (1989) 39.

[27] L.S. Brown and G. Gabrielse, Rev. of Mod. Phys. 58 (1986) 233.

[28] H.-J. Kluge et.al., Phys. Rev. C46 (1992) R2140. 

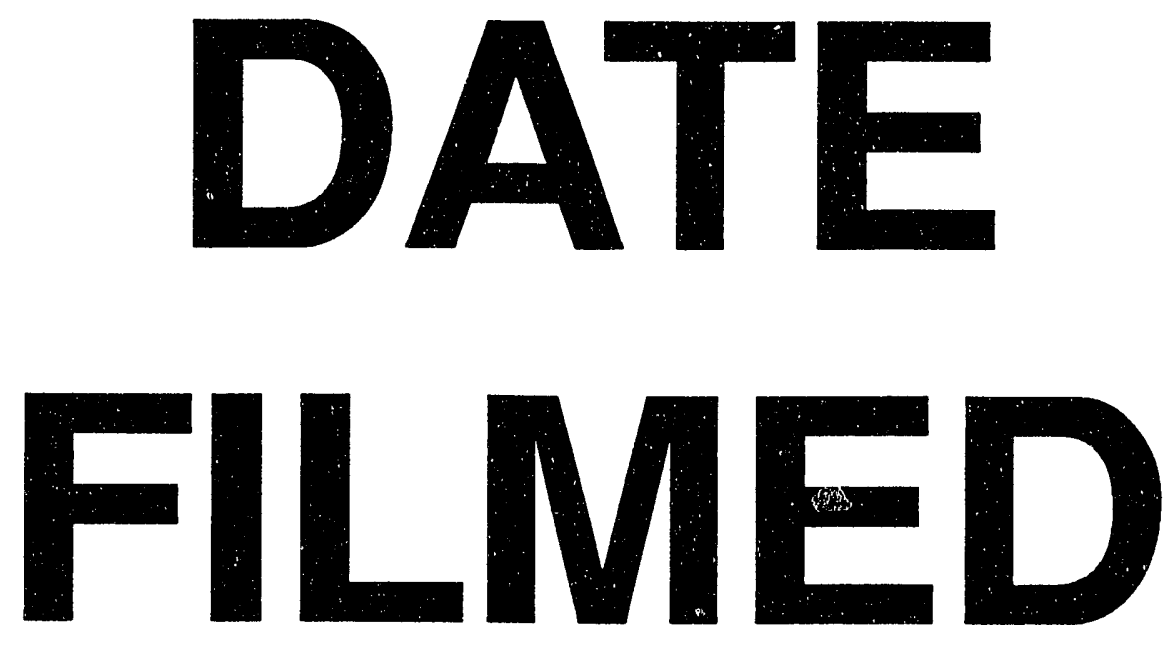

$6 / 10 / 94$
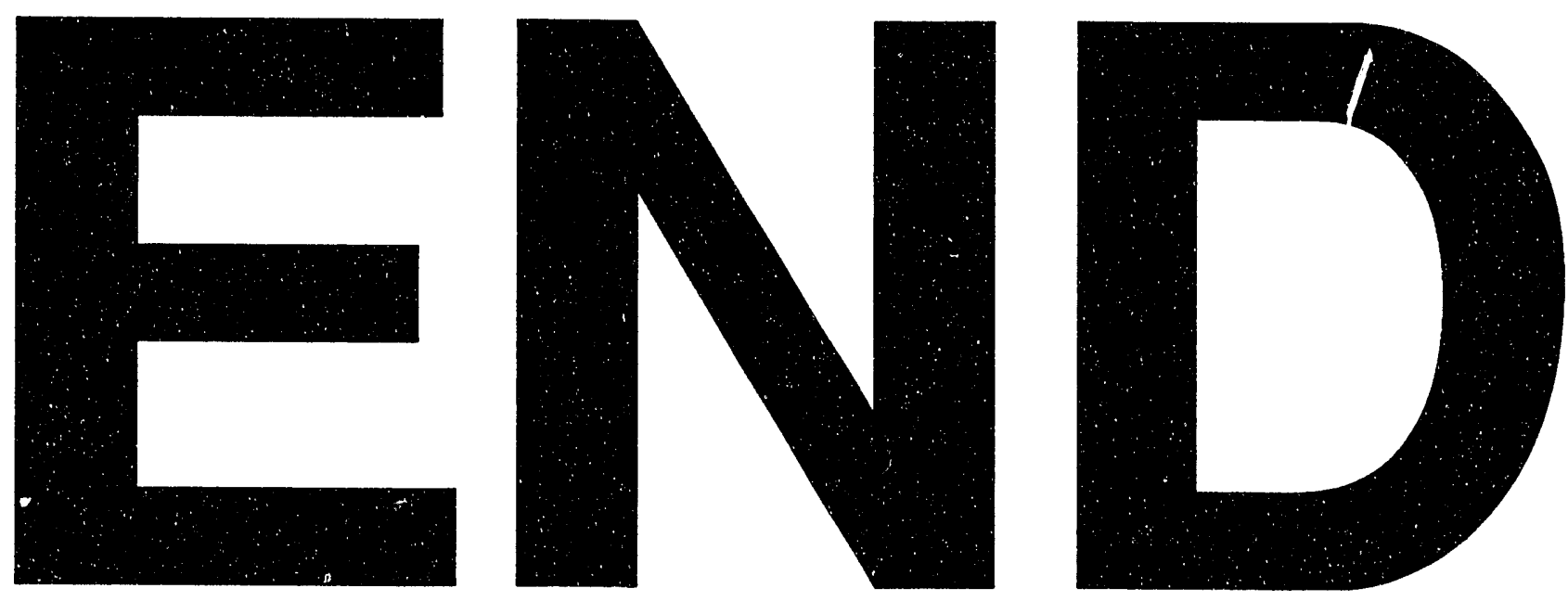
